\title{
LINEARIZATION OF CERTAIN NONLINEAR FUNCTIONAL EQUATIONS
}

\author{
R. H. CAMERON, B. W. LINDGREN, AND W. T. MARTIN
}

1. Introduction. We denote by $C$ the space of all real-valued functions $x(t)$ which are continuous for $0 \leqq t \leqq 1$ and which vanish at $t=0$. Let $T$ be the (possibly nonlinear) transformation

$$
y(t)=T x(t)=x(t)+\Lambda(x \mid t)
$$

defined over some Wiener-measurable subset $\Gamma$ of $C$ and having the property that $T$ takes $\Gamma$ into a set $T \Gamma$ in a one-one manner.

In an earlier paper [1], ${ }^{1}$ two of the present authors have studied the behavior of Wiener integrals over $\Gamma$ under one-one transformations of the form (1) which possess certain smoothness properties. In another paper [3], they have shown (for the case $\Gamma=C$ ) that the pre-image, $x(t)=T^{-1} y(t)$, can be expressed in terms of $y(t)$ as a limit in the mean of a series of "Fourier-Hermite" functionals.

In this note we wish to indicate a minimizing process which will produce approximate solutions of (1) in terms of simpler Wiener integrals. These approximate solutions approach the true solution in the sense of the $L_{1}(C)$ limit in the mean.

Specifically, these approximate solutions are obtained as follows. Let $\left\{a_{k}(s)\right\}$ be any sequence of functions closed in $L_{2}[0,1]$. Let $m$ and $p$ denote the $n$-vectors $m_{1}, \cdots, m_{n}$ and $p_{1}, \cdots, p_{n}$, and let $A_{m}^{(n)}(t)$ be a solution of the system of algebraic equations

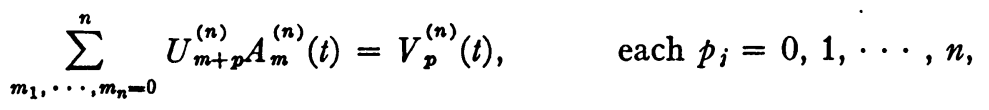

where $^{2}$

$$
U_{m}^{(n)}=\int_{\Gamma}^{w} \prod_{k=1}^{n}\left\{\int_{0}^{1} a_{k}(s) d T x(s)\right\}^{m k} d_{w} x
$$

and

$$
V_{m}^{(n)}(t)=\int_{\Gamma}^{w} x(t) \prod_{k=1}^{n}\left\{\int_{0}^{1} a_{k}(s) d T x(s)\right\}^{m_{k}} d_{w} x
$$

Presented to the Society, December 26, 1951; received by the editors May 3, 1951.

1 Numbers in brackets refer to the bibliography at the end.

2 If the functions $\left\{a_{k}(s)\right\}$ are of bounded variation on $[0,1]$, the Stieltjes integrals can be interpreted throughout as Riemann-Stieltjes integrals; otherwise (if they are merely of $\left.L_{2}[0,1]\right)$ the integrals are Paley-Wiener-Zygmund integrals [5]. 
It will be shown that under suitable conditions on $\Lambda(x \mid t)$

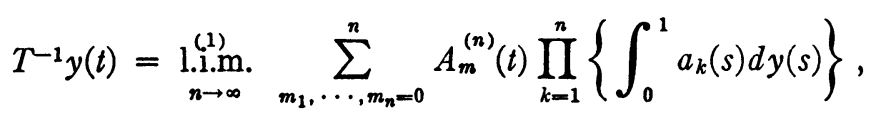

for each fixed $t$ on $[0,1]$ (where the li.m. is taken in the sense of $\left.L_{1}(T \Gamma)\right)$. The linear equations giving $A_{m}^{(n)}(t)$ will necessarily be consistent.

The conditions which we wish to impose on the transformation $T$ are precisely those of Theorem V of [1]. Since they are lengthy and are stated there explicitly, we shall not repeat them here. However, in the formulation of our result we shall use three functionals introduced in [1] related to $\Lambda(x \mid t)$; we define these here, using the notation of [1]. Let

$$
\Phi(x)=\int_{0}^{1}\left[\frac{\partial}{\partial t} \Lambda(x \mid t)\right]^{2} d t+2 \int_{0}^{1}\left[\frac{\partial}{\partial t} \Lambda(x \mid t)\right] d x(t) .
$$

Let

$$
K(x \mid t, s)
$$

be the Volterra derivative of $\Lambda(x \mid t)$, that is, a functional such that

$$
\left.\frac{\partial}{\partial h} \Lambda(x+h z \mid t)\right]_{h=0}=\int_{0}^{1} K(x \mid t, s) z(s) d s
$$

holds for $x \in \Gamma, 0 \leqq t \leqq 1$, and $z(t) \in C$. And let $D(x)$ be the Fredholm determinant of $K(x \mid t, s)$ :

$D(x)=1+\sum_{n=1}^{\infty} \frac{1}{n !} \int_{0}^{1} \cdots \int_{0}^{1}\left|\begin{array}{c}K\left(x \mid s_{1}, s_{1}\right) \cdots K\left(x \mid s_{1}, s_{n}\right) \\ \vdots \\ K\left(x \mid s_{n}, s_{1}\right) \cdots K\left(x \mid s_{n}, s_{n}\right)\end{array}\right| d s_{1} \cdots d s_{n}$.

2. The linearization theorem. We are now in a position to state our theorem.

THEOREM. Let $T$, given by (1), be a transformation which satisfies the conditions in Theorem $\mathrm{V}$ of [1]. Further, let it have the properties that $D^{2}(x) \exp \{-2 \Phi(x)\}$ and $|D(x)|^{-1} \exp \{\Phi(x)\}$ both be summable on $\Gamma$. Let $\left\{a_{k}(t)\right\}$ be any closed set of real functions in $L_{2}[0,1]$. For fixed $t$ on $[0,1]$ and for each positive integer $n$ let the constants $A_{m_{1}, \cdots, m_{n}}^{(n)}(t)$ be determined so as to minimize

(2) $\int_{\Gamma}^{w}\left[x(t)-\sum_{m_{1}, \cdots, m_{n}=0}^{n} A_{m_{1}}^{(n)}, \cdots, m_{n}(t) \prod_{k=1}^{n}\left\{\int_{0}^{1} a_{k}(s) d T x(s)\right\}^{m_{k}}\right]^{2} d_{w} x$. 
Then for this value of $t$ it follows that

$$
\begin{aligned}
& \lim _{n \rightarrow \infty} \int_{T \Gamma}^{w} \mid T^{-1} y(t)-\sum_{m_{1}, \cdots, m_{n}=0}^{n} A_{m_{1}, \cdots, m_{n}}^{(n)}(t) \\
& \cdot \prod_{k=1}^{n}\left\{\int_{0}^{1} a_{k}(s) d y(s)\right\}^{m_{k}} \mid d_{w} y=0 .
\end{aligned}
$$

In proving this theorem we shall make use of two results: first, that the functionals

$$
\prod_{k=1}^{n}\left\{\int_{0}^{1} a_{k}(s) d x(s)\right\}^{m_{k}}, \quad m_{k}=0, \cdots, n, n=1,2, \cdots,
$$

are closed in the $L_{4}$ sense over $C$; and second, Theorem $\mathrm{V}$ of [1], the conclusion of which states that if $F[y]$ is any Wiener-measurable functional on $T \Gamma$ for which either member of the equation below exists, then the other member also exists, and they are equal:

$$
\int_{T \Gamma}^{w} F[y] d_{w} y=\int_{\Gamma}^{w} F[x+\Lambda(x \mid \cdot)] e^{-\Phi(x)}|D(x)| d_{w} x .
$$

An outline of the proof of the first of these results will be given following the proof in the next section.

3. Proof of the theorem. We notice first that the functional $T^{-l} y(t)$ belongs, for each $t$, to $L_{4}(T \Gamma)$; for, applying (5), we have from Schwarz' inequality

$$
\begin{aligned}
\left(\int_{T \Gamma}^{w}\left[T^{-1} y(t)\right]^{4} d_{w} y\right)^{2} & =\left(\int_{\Gamma}^{w}[x(t)]^{4} e^{-\Phi(x)}|D(x)| d_{w} x\right)^{2} \\
& \leqq \int_{\Gamma}^{w}[x(t)]^{8} d_{w} x \int_{\Gamma}^{w} e^{-2 \Phi(x)}|D(x)|^{2} d_{w} x,
\end{aligned}
$$

and $\int_{\Gamma}^{w}[x(t)]^{8} d_{w} x$ is known to be finite for all $t$ in $[0,1]$. Then by the closure of the functionals (4) in $L_{4}(T \Gamma)$, there exist-for given $\epsilon>0$ and each $t$-an integer $N$ and constants $B_{m}^{(n)}(t)$ such that for $n \geqq N$,

$$
\begin{aligned}
\int_{T \Gamma}^{w}\left[T^{-1} y(t)-\sum_{m_{1}, \cdots, m_{n}=0}^{n} B_{m_{1}}^{(n)} \cdots, m_{n}(t)\right. & \\
& \left.\cdot \prod_{k=1}^{n}\left\{\int_{0}^{1} a_{k}(s) d y(s)\right\}^{m_{k}}\right]^{4} d_{w} y<\epsilon .
\end{aligned}
$$

Applying now (5) to the integral in (6), we obtain 


$$
\begin{aligned}
& \int_{\Gamma}^{w}\left[x(t)-\sum_{m_{1}, \cdots, m_{n}=0}^{n} B_{m_{1}}^{(n)}, \cdots, m_{n}(t) \prod_{k=1}^{n}\left\{\int_{0}^{1} a_{k}(s) d T x(s)\right\}^{m_{k}}\right]^{4} \\
& \cdot e^{-\Phi(x)}|D(x)| d_{w} x<\epsilon \text {. }
\end{aligned}
$$

Then from Schwarz' inequality we have

$$
\begin{aligned}
\int_{\Gamma}^{w}\left[x(t)-\sum_{m_{1}, \cdots, m_{n}=0}^{n} B_{m_{1}, \cdots, m_{n}}^{(n)}(t) \prod_{k=1}^{n}\left\{\int_{0}^{1} a_{k}(s) d T x(s)\right\}^{m_{k}}\right]^{2} & d_{w} x \\
& <Q_{1} \epsilon^{1 / 2}
\end{aligned}
$$

where

$$
Q_{1}^{2}=\int_{\Gamma}^{w} e^{\Phi(x)}|D(x)|^{-1} d_{w} x<\infty
$$

Now since the functions $A_{m}^{(n)}(t)$ were chosen to minimize (2), it follows that (2) is also less than $Q_{1} \epsilon^{1 / 2}$. If we make the change of variable $y=T x$ in

$$
\begin{array}{r}
\int_{T \Gamma}^{w}\left[T^{-1} y(t)-\sum_{m_{1}}, \cdots, m_{n}=0\right. \\
A_{m_{1}}, \cdots, m_{n} \\
\left.(n) \prod_{k=1}^{n}\left\{\int_{0}^{1} a_{k}(s) d y(s)\right\}^{m_{k}}\right]^{2} \\
\cdot e^{\Phi\left(T^{-1} y\right)\left|D\left(T^{-1} y\right)\right|^{-1} d_{w} y,}
\end{array}
$$

using (5), we see that (7) is equal to (2), and that hence (7) is less than $Q_{1} \epsilon^{1 / 2}$. Once again employing Schwarz' inequality, we have

$$
\begin{array}{r}
\int_{T \Gamma}^{w}\left|T^{-1} y(t)-\sum_{m_{1}, \cdots, m_{n}=0}^{n} A_{m_{1}}^{(n)}, \cdots, m_{n}(t) \prod_{k=1}^{n}\left\{\int_{0}^{1} a_{k}(s) d y(s)\right\}^{m_{k}}\right| d_{w} y \\
<Q_{2}\left(Q_{1} \epsilon^{1 / 2}\right)^{1 / 2}
\end{array}
$$

where

$$
Q_{2}^{2}=\int_{T \Gamma}^{w} e^{-\Phi\left(T^{-1} y\right)}\left|D\left(T^{-1} y\right)\right| d_{w} y .
$$

But then using (5) again, we see that $Q_{2}^{2}=\int_{\Gamma}^{w} e^{-2 \Phi(x)}|D(x)|{ }^{2} d_{w} x$, which we are assuming to be finite. Thus (3) is established.

We discuss next the closure of (4). We assume that the $\left\{a_{k}(t)\right\}$ are orthonormal as well as closed. The Schmidt process shows that this involves no loss of generality. It was shown in [2] that the "Fourier-Hermite" sequence

$$
\left\{\prod_{k=1}^{n} H_{m_{k}}\left\{\int_{0}^{1} a_{k}(t) d x(t)\right\}\right\}
$$


is closed in $L_{2}(C)$. Nothing essentially new is involved in the proof that (4) is closed in $L_{p}(T \Gamma)$ for $p>1$. We give here only a brief indication of how this closure can be established.

It is known ${ }^{3}$ that if $\left\{H_{k}(u)\right\}$ are the Hermite polynomials, then for $p>1$ the sequence $\left\{e^{-u^{2} / p} H_{k}(u)\right\}$ is complete in relation to $L_{p^{\prime}}(-\infty, \infty)$, where $1 / p+1 / p^{\prime}=1$. Induction based on the Fubini theorem shows that the sequence

$$
\left\{\prod_{k=1}^{n} H_{m_{k}}\left(u_{k}\right) \exp \left(-u_{k}^{2} / p\right)\right\}, \quad m_{1}, \cdots, m_{n}=0,1, \cdots,
$$

is complete in relation to $L_{p^{\prime}}(-\infty, \infty)^{n}$. But this completeness implies $^{4}$ closure of (9) in $L_{p}(-\infty, \infty)^{n}$. The method of [2] in showing closure in $L_{2}(C)$ of $(8)$ can then be carried over essentially unchanged to show closure of $(8)$ in $L_{p}(C)$. Finally, since $H_{k}(u)$ is a polynomial, the closure of (4) becomes evident upon rearranging the terms in the approximating linear combinations of Fourier-Hermite functionals.

REMARK. In the theorem the constants $\left\{A_{m}^{(n)}\right\}$ were determined so as to minimize (2). We may, if we wish, alter this procedure and determine constants $\left\{C_{m}^{(n)}(t)\right\}$ for each $t$ so as to minimize

$$
\begin{aligned}
& \int_{\Gamma}^{w}\left[\Lambda\left(\left.x\right|^{3} t\right)+\sum_{m_{1}, \cdots, m_{n}=0}^{n} C_{m_{1}, \cdots, m_{n}}^{(n)}(t)\right. \\
&\left.\cdot \prod_{k=1}^{n}\left\{\int_{0}^{1} a_{k}(s) d T x(s)\right\}^{m_{k}}\right]^{2} d_{w} x .
\end{aligned}
$$

Then the same argument used for (3) would show that

$$
\begin{aligned}
\lim _{n \rightarrow \infty} & \int_{T \Gamma}^{w} \mid T^{-1} y(t)-y(t) \\
& -\sum_{m_{1}, \cdots, m_{n}=0}^{n} C_{m_{1}, \cdots, m_{n}}^{(n)}(t) \prod_{k=1}^{n}\left\{\int_{0}^{1} a_{k}(s) d y(s)\right\}^{m_{k}} \mid d_{w} y=0 .
\end{aligned}
$$

Since by hypothesis $\Lambda(x \mid t)$ is a smoother functional than $x(t)$, it may sometimes be more convenient to use $\left(2^{\prime}\right)$ and $\left(3^{\prime}\right)$ than (2) and (3). On the basis of our experience in [3] it seems likely that the convergence of $\left(3^{\prime}\right)$ will in general be more rapid than that of (3).

\section{REFERENCES}

1. R. H. Cameron and W. T. Martin, The transformation of Wiener integrals by non-linear transformations, Trans. Amer. Math. Soc. vol. 66 (1949) pp. 253-283.

${ }^{3}$ See $[4$, p. 280$]$.

4 Following, for example, the argument given for one dimension in $[4$, p. 200]. 
2. - The orthogonal development of non-linear functionals in series of FourierHermite functionals, Ann. of Math. vol. 48 (1947) pp. 385-392. 642.

3. - Non-linear integral equations, Ann. of Math. vol. 51 (1950) pp. 629-

4. S. Kaczmarz and H. Steinhaus, Theorie der Orthogonalreihen, Warsaw-Lwow, 1935.

5. R. E. A. C. Paley, N. Wiener, and A. Zygmund, Notes on random functions, Math. Zeit. vol. 37 (1933) pp. 647-668.

UNIVERSITY OF MINNESOTA AND

MassachusetTs Institute of TeChNology

\section{THE MULTIPLIER RULE FOR ORDINARY DIFFERENTIAL EQUATIONS}

\section{PHILIP COOPERMAN}

One of the unsolved problems of the calculus of variations is to find a proof of the multiplier rule for the case of partial differential equations as side conditions. The essential difficulty lies in the fact that the theory of partial differential equations is not sufficiently developed to allow the use of the same proecedure which worked in the case of ordinary differential equations. Hence, it would be desirable to have a proof of the multiplier rule which made no appeal to the theory of differential equations, even in the case of ordinary differential equations. A proof of this type is the object of this paper.

Let us consider the problem of making stationary the functional $J[y]$ defined by

$$
J[y]=\int_{x_{0}}^{x_{1}} F\left(x, y_{1} \cdots y_{n}, y_{1}^{\prime} \cdots y_{n}^{\prime}\right) d x
$$

under boundary conditions which need not be specified here and under side conditions

$$
G_{j}\left(x, y_{1} \cdots y_{n}, y_{1}^{\prime} \cdots y_{n}^{\prime}\right)=0, \quad j=1, \cdots, p<n .
$$

The usual proof of the multiplier rule starts from this problem and shows that the problem of making stationary the functional $K[y, \lambda]$,

$$
K[y, \lambda]=\int_{x_{0}}^{x_{1}}\left\{F+\lambda_{j} G_{j}\right\} d x
$$

Received by the editors May 16, 1951. 